\title{
Effects of Undaria pinnatifida (wakame) on the human intestinal
}

\section{environment}

\author{
Keiko Yoshinaga ${ }^{1}$, Reiko Maruya ${ }^{2}$, Takashi Koikeda ${ }^{3}$, Takahisa Nakano ${ }^{1}$ \\ ${ }^{1}$ Riken Vitamin Co., Ltd., 2-9-18 Kanda-Misakicho, Chiyoda-ku, Tokyo, Japan; ${ }^{2}$ SOUKEN \\ Co. Ltd., 1-9-10 Hamamatsu-cho, Minato-ku, Tokyo, Japan; ${ }^{3}$ Shiba Palace Clinic, 1-9-10 \\ Hamamatsu-cho, Minato-ku, Tokyo, Japan
}

Corresponding author: Keiko Yoshinaga, PhD, Riken Vitamin Co., Ltd., 2-9-18 KandaMisakicho, Chiyoda-ku, Tokyo 101-8370, Japan

Submission Date: August $21^{\text {st }}, 2018$, Acceptance Date: October $28^{\text {th }}, 2018$, Publication Date: October $30^{\text {th }}, 2018$

Citation: Yoshinaga K., Maruya R., Koikeda T., Nakano T. Effects of Undaria pinnatifida (wakame) on the human intestinal environment. Functional Foods in Health and Disease 2018; 8(10): 488-504. DOI: https://doi.org/10.31989/ffhd.v8i10.543

\begin{abstract}
Background: Undaria pinnatifida (wakame) is an edible seaweed that is a common constituent in the Japanese diet and exhibits various biological effects. Wakame is rich in dietary fiber. Despite the long history of its intake, changes in the intestinal environment following the ingestion of wakame are unclear. The aim of this study is to investigate the daily consumption of wakame and test the possibility of wakame use as a probiotic.
\end{abstract}

Methods: We examined the effect of a 2-week intake of wakame on defecation frequency and the intestinal microbiota of 22 healthy individuals suffering from low defecation frequency. The clinical trial was designed as an open-label study.

Results: Defecation frequency, defined in terms of times per week, days per week, and volume per week, significantly increased during the wakame intake period. Furthermore, based on 
terminal restriction fragment length polymorphism (T-RFLP), the fraction of bifidobacteria as a percentage of all fecal bacteria increased significantly during the wakame intake period. At the phylum, next-generation sequencing (NGS) revealed that the relative abundance of Actinobacteria after wakame intake significantly increased while the abundance of Bacteroidetes decreased. Moreover, species-level analyses revealed that the abundance of Bifidobacterium longum increased significantly after wakame intake. B. longum colony counts on wakame-containing medium were significantly higher than those on medium lacking wakame.

Conclusion: These observations suggest that wakame intake improves intestinal environment and increases the fecal population of bifidobacteria, indicating that it may have prebiotic properties.

Keywords: Undaria pinnatifida; wakame; bowel movement; intestinal microbiota; fiber; Bifidobacterium

\section{BACKGROUND}

According to the archaeological evidence from Japan, seaweed has been part of the human diet for thousands of years. Tax records from the eighth century list seaweeds as payment to the Japanese government, indicating that they played an important role in Japanese culture. The brown seaweed Undaria pinnatifida (wakame) is rich in several minerals and vitamins, and dietary fiber, and is one of the most popular seaweeds in the traditional Japanese diet. In Japan, wakame is used in noodle dishes, soups, salads, pickles, and others [1].

Wakame exhibits various biological effects, including antihypertensive effects [2-4]. It also increases fecal dioxin excretion [5], has anticancer [6] and antiviral properties [7], and changes glucose and lipid metabolism [8]. In published clinical trials, wakame ingestion was associated with beneficial effects, as a supplemental regimen for the prevention of metabolic syndrome [9-11]. The Japanese people have the lowest incidence of metabolic syndrome and the longest life expectancy in the world. Wakame, as a component of the traditional Japanese diet, may constitute a potentially effective and safe approach for the prevention or attenuation of the metabolic syndrome.

The intestinal environment is important for human health. The microbiota composition of the human gut changes with age, and alterations of its composition influence human health [12]. Many recent reports demonstrated the importance of the intestinal environment for human 
health, particularly the link between metabolic syndrome and human gut microbiota [13]. Recent studies demonstrate the prebiotic potential of edible seaweed and its components, such as polysaccharides fucoidan and $\beta$-glucan, which interact with the human gut microbiota in vitro [14-16]. Zhang et al. (2018) reported significant structural changes in fecal microbiota of mice fed with Porphyra haitanesisi and Ulwa prolifera, which are edible seaweeds [17]. Moreover, comparative gut metagenome analyses revealed that porphyranase and agarase genes, which encode enzymes that specifically degrade sulfated polysaccharides of marine algae, frequently occur in the Japanese population but are absent from the metagenomes of North American individuals [18]. On the basis of these reports, seaweed consumption appears to be associated with changes in the gut's bacterial environment.

Despite the long history of its intake, changes in the intestinal environment following the ingestion of wakame are unclear. Moreover, the effect of a long-term (over 2 wk) wakame ingestion on the frequency of defecation and on the composition of intestinal bifidobacteria has not been studied in detail. Hence, in the current study, the intestine-conditioning effect of wakame was evaluated in healthy adults with a tendency of constipation, by comparing the frequency of bowel movements, stool quantity and quality, and the composition of the intestinal microbiota before and after wakame intake. We also examined the possibility of using wakame as a prebiotic.

\section{MATERIALS AND METHODS}

\section{Materials}

Dried wakame (product name: FUERU WAKAME-CHAN® Sanriku), manufactured by Riken Food Co., Ltd., (Tokyo, Japan) was used; $4 \mathrm{~g}$ of dried wakame contains $1.42 \mathrm{~g}$ of fiber, $0.82 \mathrm{~g}$ of protein, $0.20 \mathrm{~g}$ of lipids, and $1.58 \mathrm{~g}$ of carbohydrates.

\section{Subjects}

The subjects were 22 healthy Japanese women aged 20 to 49 years, selected from among paid volunteers recruited by SOUKEN Co., Ltd. (Tokyo, Japan). They were all registered in the SOUKEN databank of untreated subjects, who, in a questionnaire filled out in advance of the study, declared that the frequency of their bowel movements was 3-5 times/wk. Individuals with possible food allergies, alcoholics, or ones with serious diseases were excluded. Individuals periodically taking intestine-conditioning drugs, and regular users of drugs that might have an intestine-conditioning effect, were also excluded. Consequently, 37 individuals who fulfilled the criterion of low frequency of bowel movements underwent further screening 
to select the 22 study subjects.

The frequency of bowel movements of the 37 prospective subjects was evaluated based on their journal entries over the course of $1 \mathrm{wk}$, and their stool microbiota was evaluated in detail. Finally, 22 individuals (mean age, $40 \pm 6.8$ years) who fulfilled the criterion of low percentage of bifidobacteria in fecal bacteria were enrolled in the study.

\section{Study design}

The present clinical trial was designed as an open-label study to validate the effect of wakame consumption on the intestinal environment and bowel movements. The current study was approved by the Ethics Committee of the Shiba Palace Clinic and was conducted in accordance with the Declaration of Helsinki. The subjects were fully informed as to the content and method of the study, and provided written informed consent prior to the study.

The wakame intake schedule is shown in Figure 1. After a 2 wk pre-intake period, each volunteer was administered wakame for $2 \mathrm{wk}$ ( $4 \mathrm{~g}$ of dried wakame per day). The subjects were allowed to prepare it as per personal preference.

With respect to the general food intake, the subjects were asked to observe their habitual diet. Each subject reported each meal, and average daily nutritional intake per subject was calculated using Excel Eiyokun Ver.3.0. However, because the studied substance very likely affected the gut flora, other food products with similar effects were prohibited during the course of the study. During the entire study period, the subjects filled out a daily questionnaire about their diet, prescriptions, and health status. The subjects were also asked to complete a daily stool characteristics questionnaire.

The first fecal sample was collected before the subjects began the intake of wakame. The second sample was collected after a 2 week intake of wakame.

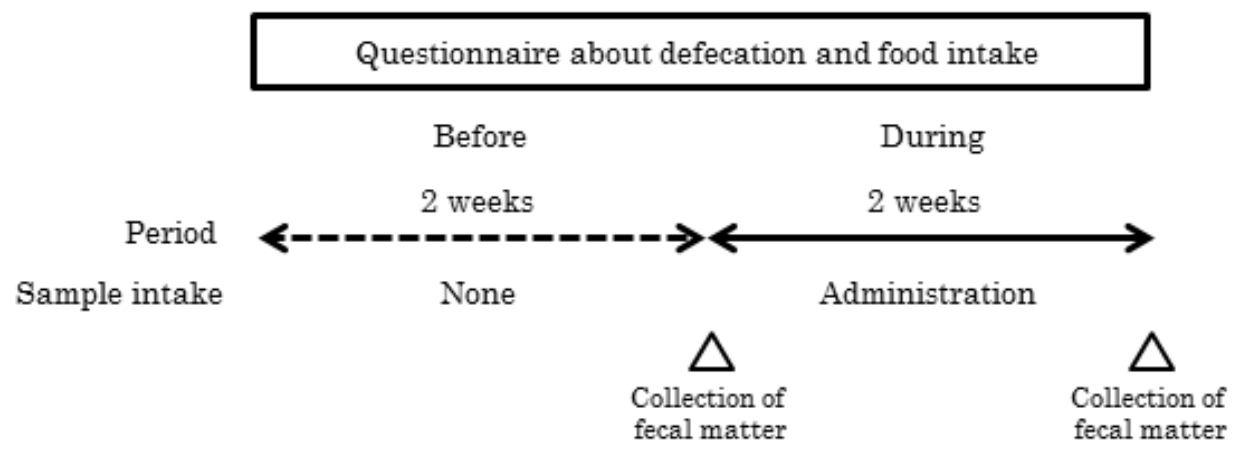

Figure 1. Study protocol 


\section{Isolation of DNA from fecal samples}

About $800 \mu \mathrm{l}$ of the fecal sample suspension preserved at $4^{\circ} \mathrm{C}$ was transferred to a tube containing zirconia beads, and the cells were disrupted using the FastPrep-24 ${ }^{\mathrm{TM}}$ 5G cell disruptor (MP Biomedicals, Irvine, CA). After cooling on ice, the samples were centrifuged at $5000 \mathrm{rpm}$ for $1 \mathrm{~min}$. DNA was automatically extracted from the processed supernatant using a 12GC and GC series Magtration-MagaZorb DNA common kit 200N (Precision System Science, Chiba, Japan). The final concentration of the extracted DNA was adjusted to $10 \mathrm{ng} / \mu \mathrm{l}$.

\section{Terminal restriction fragment length polymorphism (T-RFLP) analysis}

The terminal restriction fragment length polymorphism (T-RFLP) analysis was performed as described by Nagashima et al. (2003) [19]. Briefly, the 16S rRNA gene was amplified using the primers 516F (5'-TGCCAGCAGCCGCGGTA-3') and 1510R (5'GGTTACCTTGTTACGACTT-3'). The 5'-terminus of the forward primer (516F) was labeled with 6'-carboxyfluorescein. Amplified polymerase chain reaction (PCR) products were purified using MultiScreen ${ }^{\circledR}$ PCR $\mu 96$ filter plates (Millipore, Tokyo, Japan). The purified PCR products (about $3 \mu \mathrm{l}$ ) were digested for $3 \mathrm{~h}$ at $55^{\circ} \mathrm{C}$ with $10 \mathrm{U}$ of Bsl I restriction enzyme (New England Biolabs, Inc., Ipswich, MA). The length of the obtained fluorescent PCR fragments was determined using the ABI PRISM 3130xl genetic analyzer (Applied Biosystems, Tokyo, Japan), and the data were analyzed using the GeneMapper ${ }^{\circledR}$ software. X-rhodamine-labeled 50-1000 bp MapMarker ${ }^{\circledR}$ (BioVentures, Inc., Murfreesboro, TN) was used as a size standard.

\section{Analysis of the human fecal microbiota by next-generation sequencing (NGS)}

Sequences of the 16S rRNA gene of human fecal bacteria were analyzed by next-generation sequencing (NGS) using the MiSeq system (Illumina, San Diego, CA) as previously described Takahashi et al. (2014) [20]. The V3 and V4 hypervariable regions of the 16S rRNA gene were PCR-amplified from microbial genomic DNA using prokaryote universal primer sets: forward primer 5 '

\section{AATGATACGGCGACCACCGAGATCTACACXXXXXXXXACACTCTTTCCCTACACG} ACGCTCTTCCGATCTCCTACGGGNBGCASCAG-3 ' , where Xs represent the samplespecific 8-bp barcode sequences (CTCTCTAT, TATCCTCT, GTAAGGAG, ACTGCATA,
AAGGAGTA,
CTAAGCCT,
CGTCTAAT
and
TCTCTCCG)

and reverse primer $5^{\prime}$

CAAGCAGAAGACGGCATACGAGATZZZZZZZZGTGACTGGAGTTCAGACGTGTGC

TCTTCCGATCTGACTACNVGGGTATCTAATCC-3 ', where Zs represent the sample- 
specific 8-bp barcode sequences (TTCTGCCT, GCTCAGGA, AGGAGTCC, CATGCCTA, GTAGAGAG and CAGCCTCG); the underlined sequences represent the PCR primer region (Pro341F and Pro805R) [20] and the dual-index method [21]. The V3-V4 regions of 16S rRNA fragments were amplified in $25 \mu \mathrm{l} \mathrm{PCR}$ reactions that contained $\sim 60 \mathrm{ng}$ DNA template, 12.5 $\mu l$ 2×MightyAmp Buffer Ver.2 ( $\mathrm{Mg}^{2+}$, dNTP plus ; TaKaRa Bio, Inc. Shiga, Japan), $0.25 \mu \mathrm{M}$ each primer, and 0.625 U MightyAmp DNA polymerase (TaKaRa Bio, Inc.) [20]. The PCR reaction conditions for amplification of DNA were as follows: initial denaturation at $98{ }^{\circ} \mathrm{C}$ for $2 \mathrm{~min}$, followed by 35 cycles of annealing beginning at $65^{\circ} \mathrm{C}$ and ending at $55^{\circ} \mathrm{C}$ for $15 \mathrm{sec}$, and extension at $68^{\circ} \mathrm{C}$ for $30 \mathrm{sec}$. The annealing temperature was lowered $1{ }^{\circ} \mathrm{C}$ every cycle until it reached $55^{\circ} \mathrm{C}$, which was maintained for the remaining cycles. PCR products were purified through a MultiScreen $\mathrm{PCR}_{\mathrm{u} 96}$ filter plate (Merck Millipore, USA). To prepare amplicon pool, purified products were quantified by real-time quantitative PCR (q-PCR) on a Rotor-Gene Q quantitative thermal cycler using MightyAmp for Real-Time (SYBR Plus) [20]. Barcoded amplicons were sequenced using the MiSeq Reagent kit v.3 (a paired-end, $2 \times 284$ bp cycle on the MiSeq system (600 cycles in total)) [20].

The paired-end reads were concatenated using fastq-join with default option [22]. Only joined-reads that had quality value score of $\geq 20$ for more than $99 \%$ of the sequence were extracted using FASTX-Toolkit [20; http://hannonlab.cshl.edu/fastx_toolkit/]. Chimeric sequences were deleted with usearch6.1 [23].

Identification of bacteria from sequence reads was performed manually using the Ribosomal Database Project (RDP) Multiclassifier tool [24], which is available from the RDP website (http://rdp.cme.msu.edu/classifier/). Identification of bacterial species was performed using Metagenome@KIN Ver 2.2.1 analysis software (World Fusion, Tokyo, Japan) and the TechnoSuruga Lab Microbial Identification database DB-BA 10.0 (TechnoSuruga Laboratory, Shizuoka, Japan) with homology set at $\geq 97 \%$. Reads obtained in the FASTA format were assigned to class levels with an $80 \%$ confidence threshold.

\section{Bifidobacterium culture}

Freeze-dried pure cultures of Bifidobacterium adolescentis JCM1275, Bifidobacterium longum subsp. longum JCM1217, and Bifidobacterium bifidum JCM1255 were obtained from the Japan Collection of Microorganisms (JCM). Following initial subculturing of freeze-dried cultures, bacteria were pre-cultured in BL medium (Nissui Pharmaceutical, Tokyo, Japan) containing $5 \%$ horse blood (Kohjin Bio, Saitama, Japan). The cultures were plated on the MRS agar (CM361; Oxoid, Hampshire, UK) containing 0.8\% (Table 5) or 0-1\% (Figure 4) wakame powder, and colonies were counted after $7 \mathrm{~d}$. Rehydrated MRS was prepared according to the 
manufacturer's instructions and sterilized by autoclaving at $121^{\circ} \mathrm{C}$ for $15 \mathrm{~min}$. Transgalactosylated oligosaccharide (TOS) propionate agar (Yakult; $10 \mathrm{~g}$ of peptone, $1 \mathrm{~g}$ of yeast extract, $4.8 \mathrm{~g}$ of $\mathrm{K}_{2} \mathrm{HPO}_{4}, 3 \mathrm{~g}$ of $\mathrm{KH}_{2} \mathrm{PO}_{4}, 3 \mathrm{~g}$ of ammonium sulfate, $0.2 \mathrm{~g}$ of $\mathrm{MgSO}_{4} \cdot 7 \mathrm{H}_{2} \mathrm{O}$, $0.5 \mathrm{~g}$ of L-cysteine hydrochloride, $15 \mathrm{~g}$ of sodium propionate, $10 \mathrm{~g}$ of galacto-oligosaccharide, and $15 \mathrm{~g}$ of agar) was prepared according to the manufacturer instructions and sterilized by autoclaving at $121^{\circ} \mathrm{C}$ for $15 \mathrm{~min}$. All plates were incubated anaerobically at $37^{\circ} \mathrm{C}$ in jars, with anaerobic gas packs (Mitsubishi Gas Co., Ltd.) maintaining oxygen levels below $0.1 \%$.

\section{Statistical analysis}

The data are presented as the mean \pm standard deviation (SD). The data were analyzed using the SPSS 22.0 J software (SPSS Japan, Tokyo, Japan). The number of bowel movements, the number of days on which a bowel movement occurred, and stool volume were analyzed using Student's paired $t$-test (two-sided). Stool quality and differences in bacterial abundance were evaluated using the Wilcoxon signed-rank test. Viable counts of Bifidobacterium species were evaluated using Tukey's test. The average daily nutrient intake was analyzed using a parametric Student's paired $t$-test. $P<0.05$ was considered statistically significant.

\section{RESULTS}

\section{Food and nutrient intake}

Data for subject 1 , who took a drug because of a cold, were excluded. The average daily nutrient intake per subject during each study period is summarized in Table 1. Based on the subjects' testimonials, no significant differences in the average nutrient intake were observed except for the total fiber intake, which was significantly elevated during wakame administration.

Table 1. Average daily nutrition intake during each period

\begin{tabular}{cccccc}
\hline & \multicolumn{2}{c}{ Before intake } & Intake & $p$ value \\
\hline \hline Energy (Kcal) & $1543 \pm 269$ & $1590 \pm 258$ & 0.310 \\
Protein (gram) & $55.5 \pm 11.9$ & $57.1 \pm 11.8$ & 0.324 \\
Lipid (gram) & $55.8 \pm 11.3$ & $59.2 \pm 12.5$ & 0.127 \\
Carbohydrate (gram) & $191 \pm 33$ & $193 \pm 34$ & 0.810 \\
Colesterol (mgram) & $251 \pm 66$ & $248 \pm 75$ & 0.830 \\
Total fiber (gram) & $10.5 \pm 3$ & $11.2 \pm 2.6$ & $0.033 *$ \\
\hline
\end{tabular}

Values are expressed as the mean $\pm \mathrm{SD}(\mathrm{n}=21)$.

Significantly different from before intake period (*: $P<0.05$; Student's $t$-test). 


\section{Effect of wakame intake on frequency of bowel movements, and stool volume and quality}

The number of bowel movements, number of days with bowel movements, and stool volume significantly increased during wakame intake compared with the period before the intake (Table 2). On the other hand, the shape and color of the stool (assessed using a scoring system)

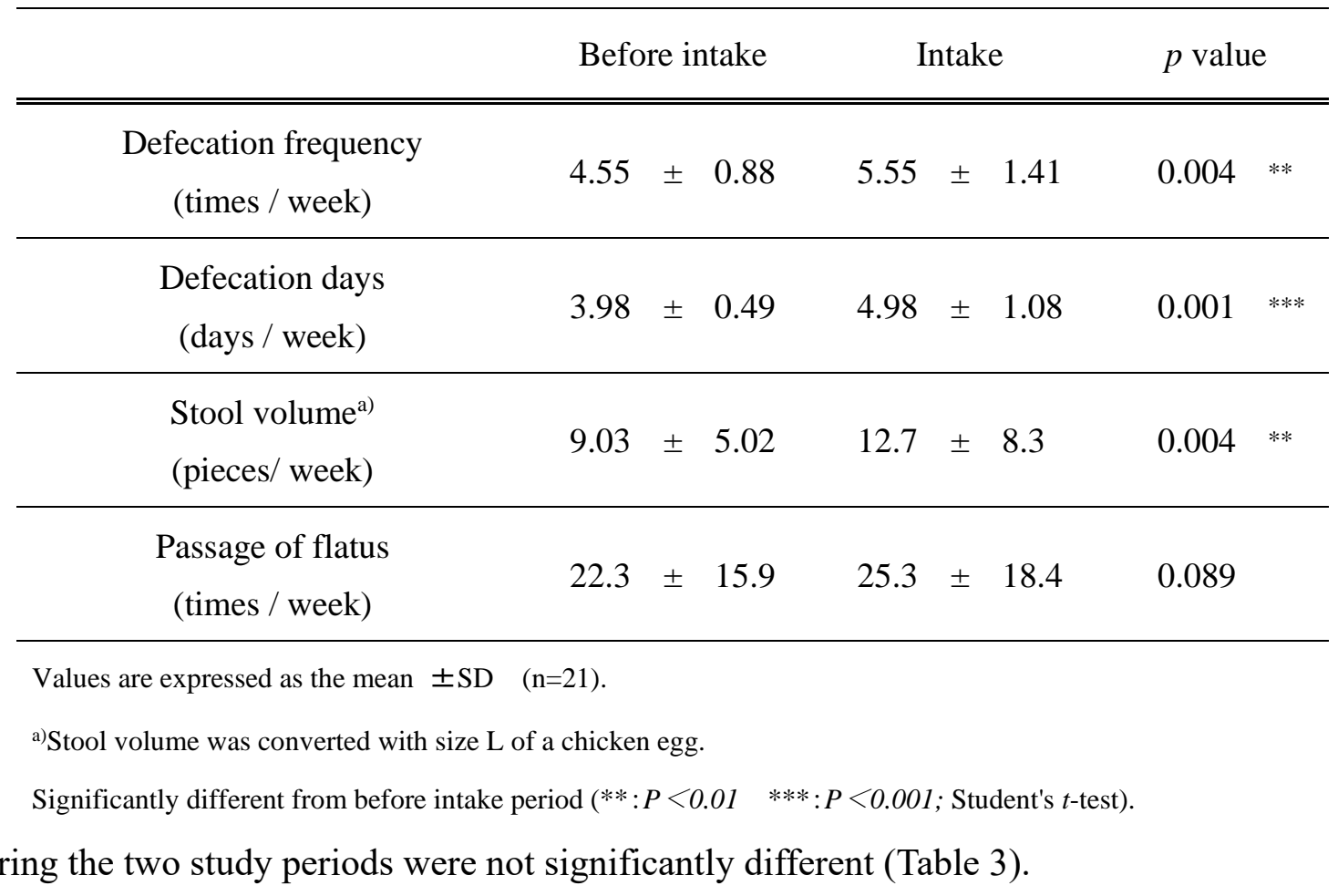

Table 2. Changes in the number of bowel movements, number of days with bowel movement, size, and passage of flatus.

Table 3. Changes in stool quality.

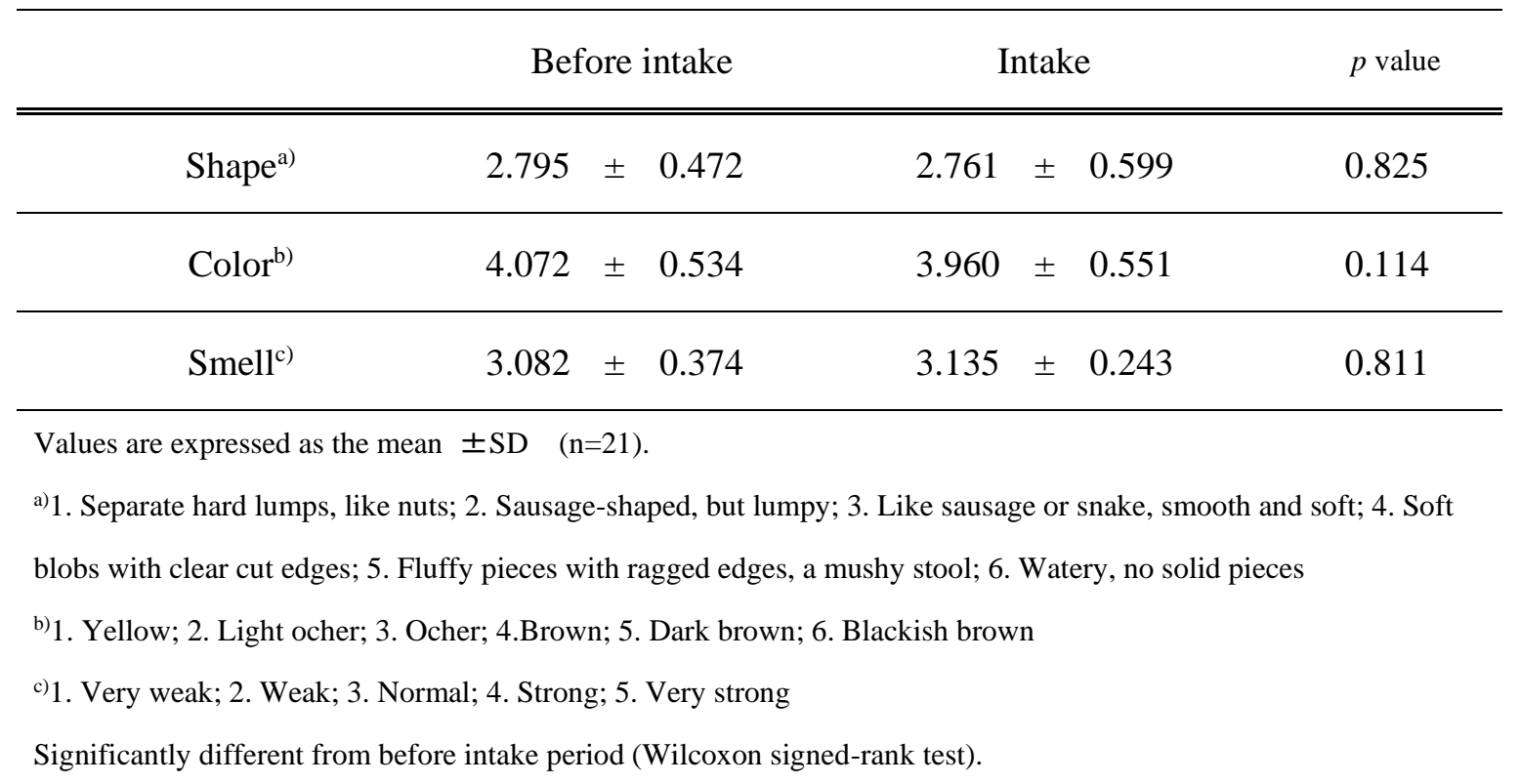




\section{The analysis of fecal microbiota}

We characterized fecal bacterial profiles according to T-RF patterns. The effect of wakame consumption on the composition of fecal microbiota is shown in Figure 2. Bifidobacterium, Lactobacillales and Clostridium cluster XVIII levels were significantly higher after wakame consumption $(P<0.05)$ than before. The increase in Bifidobacterium levels was observed in 17 out of 21 subjects ( $81 \%$ subjects). However, Bacteroides counts decreased significantly after wakame consumption $(P<0.05)$. No significant change was observed in the counts of other bacteria after wakame intake.

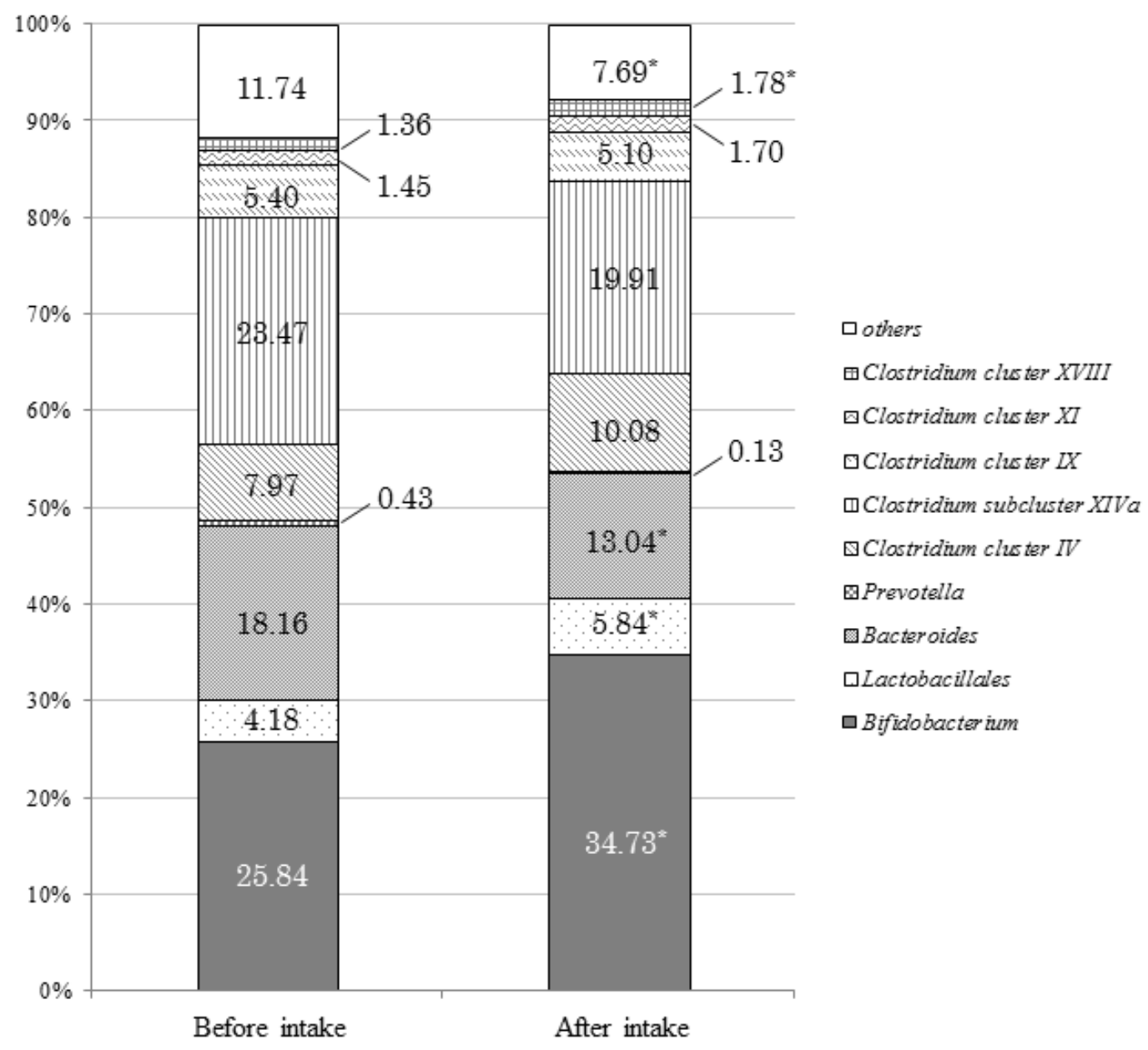

Figure 2. Bacterial profiles of each group according to T-RFLP analysis.

Significantly different from before intake period $(\mathrm{n}=21, *: P<0.05$; Wilcoxon signed-rank test)

\section{The analysis of fecal Bifidobacterium species by NGS}

We next analyzed fecal microbiota species by NGS. Figure 3 shows the changes that occurred in fecal microbiota at the phylum level. After wakame intake, the proportion of Actinobacteria increased and the proportion of Bacteroidetes decreased 
The fecal counts of Bifidobacterium species, which T-RFLP analysis showed were significantly increased after wakame intake, were examined in more detail by NGS. The effect of wakame intake on the abundance of human fecal Bifidobacterium species is shown in Table 4. Three Bifidobacterium species were detected before and after wakame intake. Bifidobacterium longum was the species whose counts were significantly increased $(P<0.05)$ after 2 wk of the wakame intake.
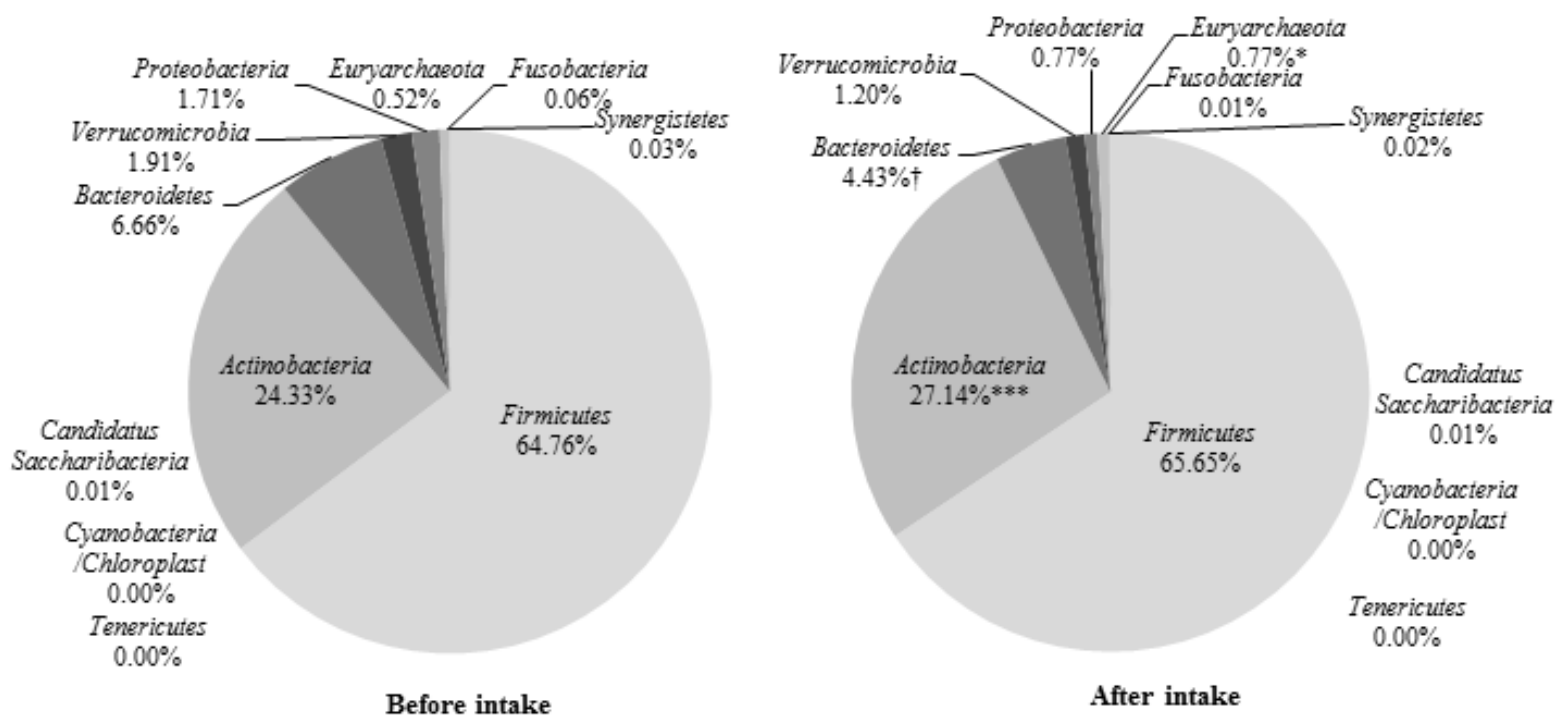

Figure 3. Average phylum distribution within the gut microbiota according to NGS analysis.

Significantly different from before intake period $(\mathrm{n}=21, *: P<0.05, * * *: P<0.001, \quad \dagger: P<0.1$; Wilcoxon signed-rank test).

Table 4. Analysis of Bifidobacterium species by NGS

\begin{tabular}{lcccccc}
\hline \multicolumn{1}{c}{ Sample } & \multicolumn{2}{c}{ Before $(\%)$} & After $(\%)$ & $p$ value \\
\hline \hline Bifidobacterium adolescentis & $6.32 \pm 6.84$ & 6.76 & \pm & 8.67 & 0.983 \\
Bifidobacterium longum & 2.45 & \pm 1.66 & 3.56 & \pm 2.52 & 0.044 \\
Bifidobacterium bifidum & 0.22 & \pm 0.45 & 0.24 & \pm & 0.56 & 0.225 \\
\hline
\end{tabular}

Values are expressed as the mean $\pm \mathrm{SD}(\mathrm{n}=21)$.

Significantly different from before intake period (*:P<0.05; Wilcoxon signed-rank test).

\section{The effect of wakame on the growth of B. longum}

Viable counts of three species of Bifidobacterium (cultured in MRS medium) are presented in

Table 5. TOS medium contains trans-galactosylated oligosaccharide and is particularly suitable for growing bifidobacteria. All speies of Bifidobacterium grew in TOS medium. B. longum and B. bifidum showed stronger growth in MRS containing wakame powder than in MRS medium 
alone. Wakame powder in MRS media did not affect the growth of $B$. adolescentis; growth was similar in MRS containing wakame powder and MRS medium alone. The effect of wakame on the growth of $B$. longum is shown in Figure 4 . After a $7 \mathrm{~d}$ culture, no $B$. longum colonies were apparent on MRS medium that did not contain wakame, but the colony counts increased with increasing wakame concentration. The colony counts of $B$. longum significantly increased in a wakame dose-dependent manner.

Table 5. Viable counts of Bifidobacterium species in different media.

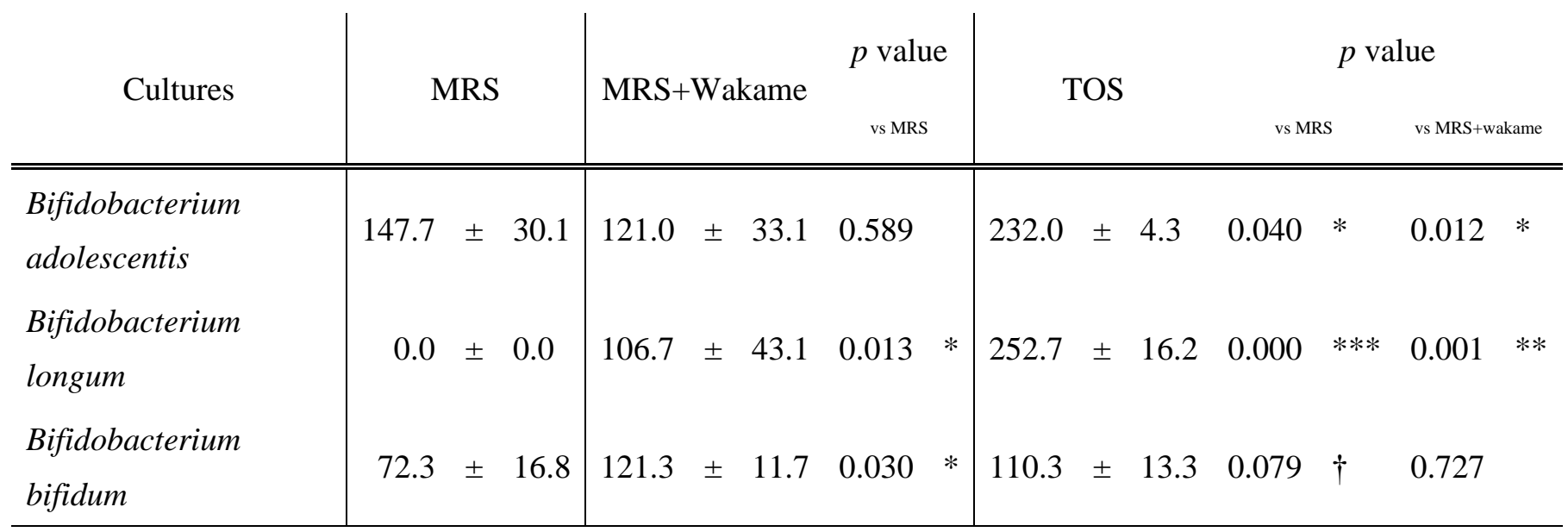

Values are expressed as the mean $\pm \mathrm{SD}$ $(n=3)$.

Significantly different from before intake period $(*: P<0.05, * *: P<0.01, * * *: P<0.001, \uparrow: P<0.1$; Turkey's test).

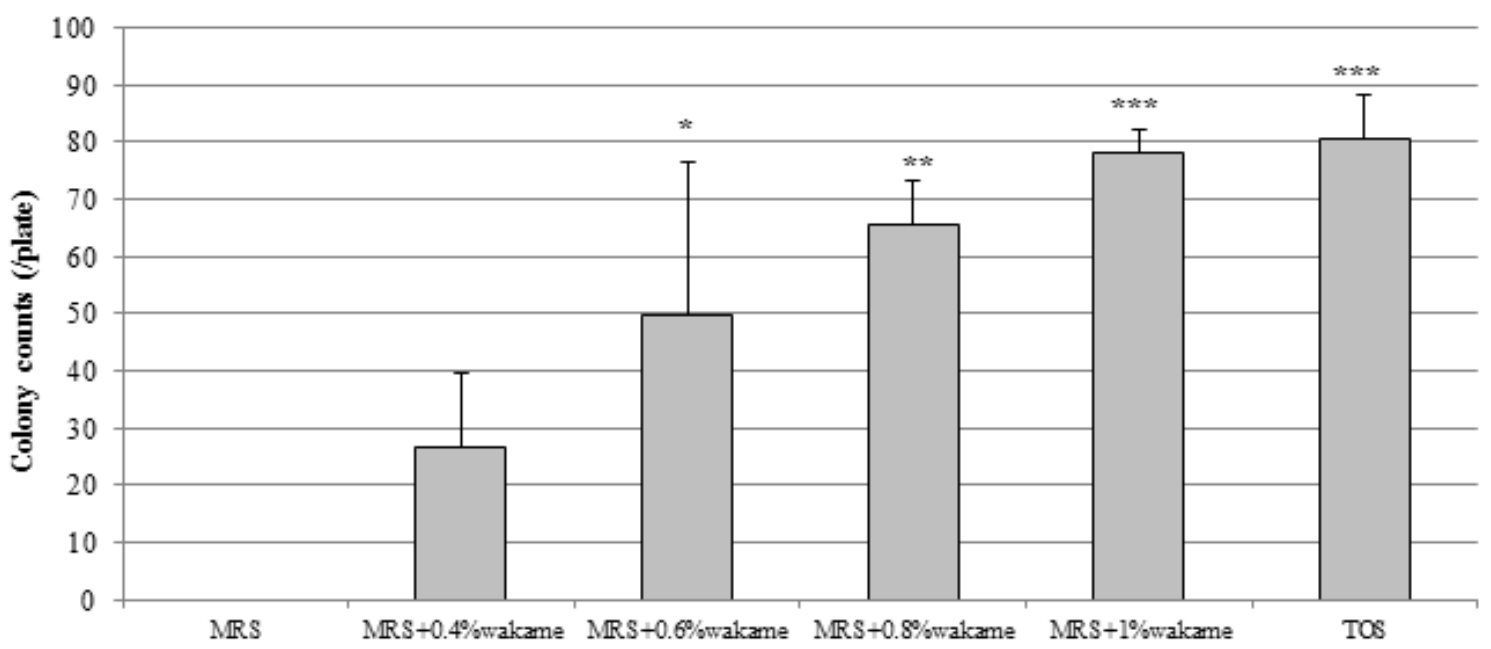

Figure 4. Bifidobacterium longum populations after 7 days of incubation with wakame powder. Values are expressed as the mean $\pm \mathrm{SD}(\mathrm{n}=3)$. Significantly different from before intake period $(*: P<0.05, * *: P<0.01, * * *: P<0.001 ;$ Tukey's test $)$. 


\section{DISCUSSION}

The current paper constitutes the first-ever report evaluating the effectiveness of seaweed in healthy subjects with a tendency for constipation, based on the state of bowel movements, stool quality, and stool microbiota composition. Stool volume and frequency of bowel movements significantly increased significantly after wakame intake (Table 2). Moreover, bifidobacterial counts in the stool increased after wakame intake (Figure 4, Table 4). These observations suggest that wakame may act like as a prebiotic.

In recent years, many people in Japan have been suffering from constipation because of the stress of daily life and intake of Western-style food. Many people who are considered healthy suffer from intestinal disorders, such as constipation, and the quality of life (QOL) is greatly associated with severity of constipation [25]. Therefore, maintaining good bowel movements and conditioning the intestinal environment are prerequisite for physical and mental health.

Pro- and prebiotics, e.g., Lactobacillus and dietary fiber, improve the bowel movements $[26,27]$. Seaweed is a rich source of dietary fibers such as arginic acid, fucoidan and laminaran [28]. Dietary fiber, in turn, positively affects the gastrointestinal health and reduces the risk of metabolic syndrome [29]. Iwata et al. reported a significant increase in the growth rate of $B$. longum in a medium containing dietary fiber from the peel of Chinese yam, pea pod, and broad bean pad [30]. Bajury et al. demonstrated a significant increase in the Bifidobacterium population after treating a $\mathrm{pH}$-controlled batch culture system inoculated with human faeces (to mimic the distal colon) with Red seaweed [14]. Terada et al. reported a significant increase in the numbers of bifidobacteria after wakame intake and a significant decrease in the amount of faecal ammonia during alginate consumption [31]. In the current study, we demonstrated an increase in B. longum counts after wakame intake and an increase in the bifidogenic growthstimulating activity of wakame in vitro (Table 4, Table 5, Figure 4). Therefore, the wakame fiber may be used by bifidobacteria. However, in the current study, the subjects were administered only $4 \mathrm{~g}$ of wakame per day, i.e., $1.4 \mathrm{~g}$ of wakame fiber per day. It has been reported that $10 \mathrm{~g}$ alginate intake once a day for 2 weeks affects faecal microbiota [31]. Thus, it is possible that another factor stimulates bifidobacterial growth upon wakame intake. According to Nutritional Intake Status Survey by National Instisute of Health and Nutrition, mean seaweeds intakes were trend to decrease year by year in Japan. Mean seaweeds was 14.6 $\pm 30.1 \mathrm{~g}$ per day in a wet form at 2002, but at 2016, 10.9 $\pm 20.1 \mathrm{~g}$ per day (www.nibiohn.go.jp/eiken/kenkounippon21/en//eiyouchousa/kekka_syokuhin_chousa_koum oku.html). A dried wakame increase tenfold by soaking. In Japan, about $3 \mathrm{~g}$ of dried wakame 
usually used to saladas, pickles, and more per plate. Therefore, intaking $4 \mathrm{~g}$ of dried wakame per day is a reasonable volume.

Bifidobacteria are natural inhabitants of the human gastrointestinal tract, and are particularly abundant in the infant gut. They exert various health-promoting effects, and consequently, their diversity and numbers are markers of the stability of human intestinal microbiota, and the intestinal environment [32]. Bifidobacteria produce organic acids, which reduce the intestinal $\mathrm{pH}$ and create an acidic environment that regulates the growth of putrefactive bacteria [33]. Woting et al. (2015) reported that the ability of oligofructose to reduce obesity and improve glucose tolerance in gnotobiotic mice fed a high-fat diet is independent of the presence of B. longum, suggesting that B. longum plays a role in metabolic syndrome [34]. In this report, we demonstrated that wakame promotes growth of B. longum (Table 4). Wakame may prevent metabolic syndrome via a prebiotic function.

The percentage of Actinobacteria, to which Bifidobacterium belongs, significantly increased and the percentage of Bacteroidetes decreased (Figure 3). Compositional changes in gut microbiota in Japanese subjects with age have been reported [12]. In their report, the relative abundance of Actinobacteria decreased with age, and an increase in the relative abundance of Bacteroidetes was observed in subjects over 70 years old. Kato-Kataoka et al. reported that the percentage of bacteria belonging to the phylum Bacteroidetes increased under the examination stress only in the placebo group compared with Lactobacillus casei-fermented milk group [35]. Wakame may affect human aging and QOL via the intestinal environment.

The human intestinal microbiota is impacted by various factors, e.g., the diet, environment, age, gender, and geographical and socioeconomic situation; among these, the diet has the most profound impact [36]. Seaweed is an important component of the daily diet in Japan, and over the centuries, seaweed has contributed to Japanese health. Prior to the present study, the ability of wakame to prevent metabolic syndrome in human has been suggested $[9,10]$. The findings of the current study suggest that wakame may help to maintain an appropriate intestinal environment.

Further studies to determine functional constituent and assessment of durability of shift in microbiome and all remain to be solved.

\section{CONCLUSION}

The present study demonstrated for the first time that daily consumption of wakame affects in healthy subjects with intestinal environment. The possibility of using wakame as a prebiotic was indicated. Wakame may help to maintain an appropriate intestinal environment. 
List of Abbreviations: JCM, Japan Collection of Microorganisms; NGS, next-generation sequencing; TOS, transgalactosylated oligosaccharide; T-RFLP, terminal restriction fragment length polymorphism

Competing Interests: There are no conflicts of interest to declare.

Authors' contributions: KY, RM, TK, and TN designed the research. KY, R.M, TK conducted the research. KY and RM performed statistical analyses. KY prepared the manuscript.

Acknowledgements and Funding: We thank T. Hisada, K. Suzuki and N. Tazato (NCIMB group, TechnoSuruga Laboratory Co., Ltd.) for their assistance with T-RFLP and NGS analyses of fecal samples, and with colony counting. This study was supported by funding from Riken Vitamin Co., Ltd., which also provided the study products for this clinical study.

\section{REFERENCES}

1. Nisizawa $K$, Noda H, Kikuchi R, Watanabe T: The main seaweed foods in Japan. Hydrobiologia 1987, 151-152: 5-29.

2. Sato M, Oba T, Yamaguchi T, Nakano T, Kahara T, Funayama K, Kobayashi A, et al.: Antihypertensive effects of hydrolysates of wakame (Undaria pinnatifida) and their angiotensin-I-converting enzyme inhibitory activity. Ann Nutr Metab 2002, 46: 259-267.

3. Sato M, Hosokawa T, Yamaguchi T, Nakano T, Muramoto K, Kahara T, Funayama K, et al.: Angiotensin I-converting enzyme inhibitory peptides derived from wakame (Undaria pinnatifida) and their antihypertensive effect in spontaneously hypertensive rats. J Agric Food Chem 2002, 50: 6245-6252.

4. Ikeda K, Kitamura A, Machida H, Watanabe M, Negishi H, Hiraoka J, Nakano T: Effect of Undaria pinnatifida (Wakame) on the development of cerebrovascular diseases in stroke-prone spontaneously hypertensive rats. Clin Exp Pharmacol Physiol 2003, 30: 44 48.

5. Morita K, Nakano T: Seaweed accelerates the excretion of dioxin stored in rats. J Agric Food Chem 2002, 50: 910-917.

6. Funahashi H, Imai T, Tanaka Y, Tsukamura K, Hayakawa Y, Kikumori T, Mase T, et al.: Wakame seaweed suppresses the proliferation of 7,12-dimethylbenz(a)-anthraceneinduced mammary tumors in rats. Jpn J Cancer Res 1999, 90: 922-927.

7. Teas J, Irhimeh MR: Dietary algae and HIV/AIDS: proof of concept clinical data. J Appl Phycol 2012, 24: 575-582. 
8. Yoshinaga $\mathrm{K}$, Nakai $\mathrm{Y}$, Izumi H, Nagaosa $\mathrm{K}$, Ishijima $\mathrm{T}$, Nakano $\mathrm{T}$, Abe K: Oral Administration of Edible Seaweed Undaria Pinnatifida (Wakame) Modifies Glucose and Lipid Metabolism in Rats: A DNA Microarray Analysis. Mol Nutr Food Res 2018, 62(12): e1700828.

9. Hata Y, Nakajima K, Uchida J, Hidaka H, Nakano T: Clinical effects of brown seaweed, Undaria pinnatifida (wakame), on blood pressure in hypertensive subjects. J Clin Biochem Nutr 2001, 30: 43-53.

10. Teas J, Baldeon ME, Chiriboga DE, Davis JR, Sarries AJ, Braverman LE: Could dietary seaweed reverse the metabolic syndrome? Asia Pac J Clin Nutr 2009, 18: 145-154.

11. Yamori Y, Miura A, Taira K: Implications from and for food cultures for cardiovascular diseases: Japanese food, particularly Okinawan diets. Asia Pac J Clin Nutr 2001, 10: 144 145.

12. Odamaki T, Kato K, Sugahara H, Hashikura N, Takahashi S, Xiao JZ, Abe F, et al.: Age - related changes in gut microbiota composition from newborn to centenarian: a crosssectional study. BMC Microbiol 2016, 16: 90.

13. Zupancic ML, Cantarel BL, Liu Z, Drabek EF, Ryan KA, Cirimotich S, Jones C, Knight R, Walters WA, Knights D, Mongodin EF, Horenstein RB, Mitchell BD, Steinle N, Snitker S, Shuldiner AR, Fraser CM: Analysis of the gut microbiota in the old order Amish and its relation to the metabolic syndrome. PLoS One 2012, 7: e43052.

14. Bajury DM, Rawi MH, Sazali IH, Abdullah A, Sarbini SR: Prebiotic evaluation of red seaweed (Kappaphycus alvarezii) using in vitro colon model. Int J Food Sci Nutr 2017, 68: $821-828$.

15. Marzorati M, Verhelst A, Luta G, Sinnott R, Verstraete W, Van de Wiele T, Possemiers S: In vitro modulation of the human gastrointestinal microbial community by plantderived polysaccharide-rich dietary supplements. Int J Food Microbiol 2010, 139: 168176.

16. Zhao J, Cheung PC: Comparative proteome analysis of Bifidobacterium longum subsp. infantis grown on $\beta$-glucans from different sources and a model for their utilization. $\mathrm{J}$ Agric Food Chem 2013, 61: 4360-4370.

17. Zhang Z, Wang X, Han S, Liu C, Liu F: Effect of two seaweed polysaccharides on intestinal microbiota in mice evaluated by illumina PE250 sequencing. Int J Biol Macromol 2018, S0141-8130: 33868-0.

18. Hehemann JH, Correc G, Barbeyron T, Helbert W, Czjzek M, Michel G: Transfer of 
carbohydrate-active enzymes from marine bacteria to Japanese gut microbiota. Nature 2010, 464: 908-912.

19. Nagashima K, Hisada T, Sato M, Mochizuki J: Application of new primer-enzyme combinations to terminal restriction fragment length polymorphism profiling of bacterial populations in human feces. Appl Environ Microbiol 2003, 69: 1251-1262.

20. Takahashi S, Tomita J, Nishioka K, Hisada T, Nishijima M: Development of a prokaryotic universal primer for simultaneous analysis of Bacteria and Archaea using next-generation sequencing. PLoS One 2014, 9: e105592.

21. Hisada T, Endoh K, Kuriki K: Inter- and intra-individual variations in seasonal and daily stabilities of the human gut microbiota in Japanese. Arch Microbiol 2015, 197: 919-934.

22. Ea-utils "Command-line tools for processing biological sequencing data" [https://expressionanalysis.github.io/ea-utils/2011]

23. Edgar RC, Haas BJ, Clemente JC, Quince C, Knight R: UCHIME improves sensitivity and speed of chimera detection. Bioinformatics 2011, 27: 2194-2200.

24. Wang Q, Garrity GM, Tiedje JM, Cole JR: Naive Bayesian classifier for rapid assignment of rRNA sequences into the new bacterial taxonomy. Appl Environ Microbiol 2007, 73: $5261-5267$.

25. Tsunoda A, Yamada K, Takano M, Kusanagi H: The translation and validation of the Japanese version of the patient assessment of constipation quality of life scale. Surg Today 2016, 46: 414-421.

26. Suzuki N, Murakoshi M, Ono T, Morishita S, Koide M, Bae MJ, Totsuka M, et al.: Effects of Enteric-coated Lactoferrin Tablets Containing Lactobacillus brevis subsp. coagulans on Fecal Properties, Defecation Frequency and Intestinal Microbiota of Japanese Women with a Tendency for Constipation: a Randomized Placebo-controlled Crossover Study. Biosci Microbiota Food Health 2013, 32: 13-21.

27. Hamaguchi N, Hirai H, Bito H, Ogawa K: Effects of Resistant Glucan Mixture on Bowel Movement in Female Volunteers. J Nutr Sci Vitaminol (Tokyo) 2016, 62: 62-66.

28. Ruperez P, Toledano G: Indigestible fraction of edible marine seaweeds. J Sci Food Agric 2003, 83: 1267-1272.

29. Thompson SV, Hannon BA, An R, Holscher HD: Effects of isolated soluble fiber supplementation on body weight, glycemia, and insulinemia in adults with overweight and obesity: a systematic review and meta-analysis of randomized controlled trials. Am J Clin Nutr 2017, 106: 1514-1528. 
30. Iwata $\mathrm{E}$, Hotta H, Goto M: The screening method of a bifidogenic dietary fiber extracted from inedible parts of vegetables. J Nutr Sci Vitaminol (Tokyo) 2009, 55: 385-388.

31. Terada A, Hara H, Mitsuoka T: Effect of dietary alginate on the faecal microbiota and faecal metabolic activity in humans. Microb Ecol Heal Dis 1995, 8: 259-266.

32. Tojo R, Suarez A, Clemente MG, De los Reyes-Gavilán CG, Margolles A, Gueimonde M, Ruas-Madiedo P: Intestinal microbiota in health and disease: role of bifidobacteria in gut homeostasis. World J Gastroenterol 2014, 20: 15163-15176.

33. Gibson GR, Wang X: Regulatory effects of bifidobacteria on the growth of other colonic bacteria. J Appl Bacteriol 1994, 77: 412-420.

34. Woting A, Pfeiffer N, Hanske L, Loh G, Klaus S, Blaut M: Alleviation of high fat dietinduced obesity by oligofructose in gnotobiotic mice is independent of presence of Bifidobacterium longum. Mol Nutr Food Res 2015, 59: 2267-2278.

35. Kato-Kataoka A, Nishida K, Takada M, Kawai M, Kikuchi-Hayakawa H, Suda K, Ishikawa H, et al.: Fermented Milk Containing Lactobacillus casei Strain Shirota Preserves the Diversity of the Gut Microbiota and Relieves Abdominal Dysfunction in Healthy Medical Students Exposed to Academic Stress. Appl Environ Microbiol 2016, 82: $3649-3658$.

36. Suzuki Y, Ikeda K, Sakuma K, Kawai S, Sawaki K, Asahara T, Takahashi T, et al.: Association between Yogurt Consumption and Intestinal Microbiota in Healthy Young Adults Differs by Host Gender. Front Microbiol 2017, 8: 847. 